\title{
Luminosity profiles of advanced mergers of galaxies using 2MASS data
}

\author{
A. Chitre and C. J. Jog
}

\author{
Department of Physics, Indian Institute of Science, Bangalore 560 012, India \\ e-mail: cjjog@physics.iisc.ernet.in \\ Received 16 November 2001 / Accepted 8 March 2002
}

\begin{abstract}
A sample of 27 disturbed galaxies that show signs of interaction but have a single nucleus were selected from the Arp and the Arp-Madore catalogues. For these, the $K_{\mathrm{s}}$ band images from the Two Micron All Sky Survey (2MASS) are analysed to obtain their radial luminosity profiles and other structural parameters. We find that in spite of their similar optical appearance, the sample galaxies vary in their dynamical properties, and fall into two distinct classes. The first class consists of galaxies which can be described by a single $r^{1 / 4}$ law and the second class consists of galaxies that show an outer exponential disk. A few galaxies that have disturbed profiles cannot be fit into either of the above classes. However, all the galaxies are similar in all other parameters such as the far-infrared colours, the molecular hydrogen content and the central velocity dispersion. Thus, the dynamical parameters of these sets seem to be determined by the ratio of the initial masses of the colliding galaxies. We propose that the galaxies in the first class result from a merger of spiral galaxies of equal masses whereas the second class of galaxies results from a merger of unequal mass galaxies. The few objects that do not fall into either category show a disturbed luminosity profile and a wandering centre, which is indicative of these being unrelaxed mergers. Of the 27 galaxies in our sample, 9 show elliptical-like profiles and 13 show an outer exponential. Interestingly, Arp 224, the second oldest merger remnant of the Toomre sequence shows an exponential disk in the outer parts.
\end{abstract}

Key words. galaxies: kinematics and dynamics - galaxies: evolution - galaxies: interactions - galaxies: photometry galaxies: structure

\section{Introduction}

It was proposed theoretically a long time ago that a merger of a pair of equal-mass spirals gives rise to an elliptical galaxy (Toomre 1977). This has been confirmed observationally by near-IR studies of a few mergers which indeed show an $r^{1 / 4}$ de Vaucouleur's radial profile typical of ellipticals (Schweizer 1982; Joseph \& Wright 1985; Wright et al. 1990; Stanford \& Bushouse 1991, SB91 hereafter). Theoretical work, involving mergers of galaxies (simulated using stars alone), has shown that under some conditions mergers indeed give rise to ellipticals with the above radial profile (Barnes \& Hernquist 1991; Hernquist 1992). The mergers simulated with star formation in gas-rich spirals (Bekki 1998a) also give a similar profile.

Despite this overall agreement, there are many issues which need to be answered. First, the origin of the $r^{1 / 4}$ profile is not physically well understood, although violent relaxation is believed to be responsible for the elliptical light distribution (Lynden-Bell 1967). Both collisionless relaxation in an isolated galaxy (van Albada 1982) as well as in a merger (e.g. Barnes 1988) are known to result in an $r^{1 / 4}$ profile. Second, it is not clear if an $r^{1 / 4}$ profile

Send offprint requests to: A. Chitre,

e-mail: aparna@physics.iisc.ernet.in over the entire galaxy is the only outcome that is possible for a galaxy merger, because the parameter space for galaxy interaction has not yet been studied exhaustively by the $N$-body theoretical work in the literature. The real galaxy mergers will presumably tell us about the parameter space that needs to be explored in future theoretical work. Third, other properties such as asymmetry in morphology, twisted isophotes, boxy/disky isophotes, shells, etc. have been shown to be good indicators that an elliptical galaxy was formed by a merger event (Nieto \& Bender 1989; Hernquist \& Spergel 1992).

In order to shed light on some of these issues, in this paper we have analyzed the near infrared $K_{\mathrm{s}}$ band images from the 2MASS database for a sample of galaxies that show evidence of an advanced merger and we have also deduced other structural parameters. The large size of the database allows us to construct a big sample. The $K_{\mathrm{s}}$ band best traces the underlying mass distribution in a galaxy. Also, the extinction in the $K_{\mathrm{s}}$ band is very low, which enables us to study the underlying stellar distribution free of the effects of dust obscuration and young star-forming regions. We have chosen a sample of colliding galaxies from the Arp (1966) and Arp-Madore (1987) catalogs based purely on their optical appearance e.g. galaxies that show strong signs of interaction such as tails and a distorted, 
puffed-up main body but have a single merged nucleus. A similar study was first done by SB91 for ten galaxies, except that they considered the profiles only along the major axis and the minor axis. The subsequent papers to look at this problem have chosen their samples from IRAS (James et al. 1999; Scoville et al. 2000; Zheng et al. 1999) and are therefore biased towards high-luminosity, distant galaxies. Our sample, on the other hand, is unbiased with respect to the IR luminosity of a galaxy.

In this paper, we extend the study of SB91 for a much larger sample of interacting galaxies, and study their radial profiles as well as other structural parameters. We give new detections of the $r^{1 / 4}$ profile in four galaxies from the Arp catalogue and thus our work has extended the known number of mergers showing such profiles. We have looked at the full azimuthal data and thus the luminosity of the galaxies is sampled in a better way. We check what fraction of our sample shows the $r^{1 / 4}$ profile expected for an elliptical galaxy and compare our results with those of the SB91, and other previous work. We also study other structural parameters such as boxiness/diskiness of isophotes, wandering centres etc. that provide important additional information on mergers. In Sect. 2, we describe the criteria for selection for our sample and also the data acquired from the public sites. In Sect. 3, we describe the data analysis. In Sect. 4, we discuss the results and our conclusions are summarized in Sect. 5 .

\section{Sample selection}

The sample galaxies were chosen from the Atlas of Peculiar Galaxies (Arp 1966) based solely on their optical appearance. Galaxies which were classified as "neither E nor S" were chosen in order to select merging systems that had already lost their individual identities but had not yet reached the final smooth stage. This class of objects from the Arp catalogue is made up of peculiar, disturbed galaxies that show various features, such as adjacent loops or irregular clumps, appearance of fission, etc. The Arp-Madore (1987) catalogue of Southern Peculiar Galaxies and Associations was also used. Galaxies lying within the categories 7 (galaxies with jets), 15 (galaxies with tails, loops of material or debris and category 16 (irregular or disturbed galaxies) were selected and only those that had 2MASS data were added to our sample. The sample was unbiased towards any other properties of the galaxies such as luminosity or spectral type. The 2MASS database at http://irsa.ipac.caltech. edu was searched and all selected galaxies present in the 2MASS extended source full resolution image server were chosen. Galaxies that showed either the presence of a double nucleus or had a diffuse appearance or poorly defined centre in the 2MASS images were rejected. Galaxies with a radius of less then $10^{\prime \prime}$ were also not considered. The final sample consists of 27 galaxies. This sample is presented in the form of contour plots in Fig. 1. Table 1 gives the sample galaxies and the columns are as follows: Col. 1: the name of the galaxy; Col. 2: the alternate name of the galaxy; Cols. 3 and 4: the RA and Dec of the galaxy; Col. 5: the type of the galaxy from RC3 and Col. 6: the type of the galaxy taken from SIMBAD.

Some basic data derived from the literature for the sample galaxies are given in Table 2 where: Col. 1: the name of the galaxy; Col. 2: the ratio of the flux at $100 \mu$ to the flux at $60 \mu$; Col. 3 : the infrared luminosity between $(8-1000 \mu)$ computed using:

$L_{\mathrm{IR}}=4 \pi D^{2} F_{\mathrm{IR}}$

where

$F_{\text {IR }}=1.8 \times 10^{-14}\left(13.48 f_{12}+5.16 f_{25}+2.58 f_{60}+f_{100}\right)(2)$

from Sanders \& Mirabel (1996); Col. 4: the absolute blue luminosity from the LEDA database (http://leda.univ-lyon1.fr/); Col. 5: the semi-major axis of the elliptical isophote at $20 \mathrm{mag} \operatorname{arcsec}^{-2}$ taken from the 2MASS database and Col. 6 : the $K_{\mathrm{s}}$ magnitude contained within the semi-major axis in Col. 5 .

\section{Analysis}

The 2MASS full-resolution images for extended sources are 3-dimensional FITS image cubes. Each cube contains three image planes, and each image plane contains the $J$, $H$ and $K_{\mathrm{s}}$ 2MASS images. The image size is scaled to the size of the object, ranging from $21^{\prime \prime} \times 21^{\prime \prime}$ to $101^{\prime \prime} \times 101^{\prime \prime}$. The 3 -D FITS image cubes containing the $J, H$ and $K_{\mathrm{s}}$ 2MASS image data were downloaded and the $K_{\mathrm{s}}$ band images were extracted from them. The $K_{\mathrm{s}}$ band images were analysed using the task ELLIPSE within STSDAS ${ }^{1}$. The procedure consisted of fitting elliptical isophotes to the $K_{\mathrm{s}}$ band galaxy images and deriving the 1-dimensional azimuthally averaged radial profiles for the surface brightness, ellipticity, position angle, etc. based on the algorithm given by Jedrejewski (1987). The centre, position angle and ellipticity were allowed to vary. Ellipses were fit right up to the central pixel. The parameters thus derived, namely the surface brightness profile, the ellipticity and the position angle variation, the coefficient $(B 4)$ of the $\cos (4 \theta)$ component and the $x$ and $y$ coordinates of the centres, were studied. Galaxies having a radius larger than $50^{\prime \prime}$ could not be studied beyond that radius due to the constraint imposed by the maximum size of $101^{\prime \prime} \times 101^{\prime \prime}$ on the full resolution images.

\subsection{Fits to the surface brightness profiles}

The 1-D surface brightness profiles obtained using the ellipse fitting technique were examined. In each case, the magnitude zero points were taken from the FITS header and applied to the profiles derived from the isophotal analysis to compute the calibrated surface brightnesses.

\footnotetext{
1 The Space Telescope Science Data Analysis System STSDAS is distributed by the Space Telescope Science Institute.
} 

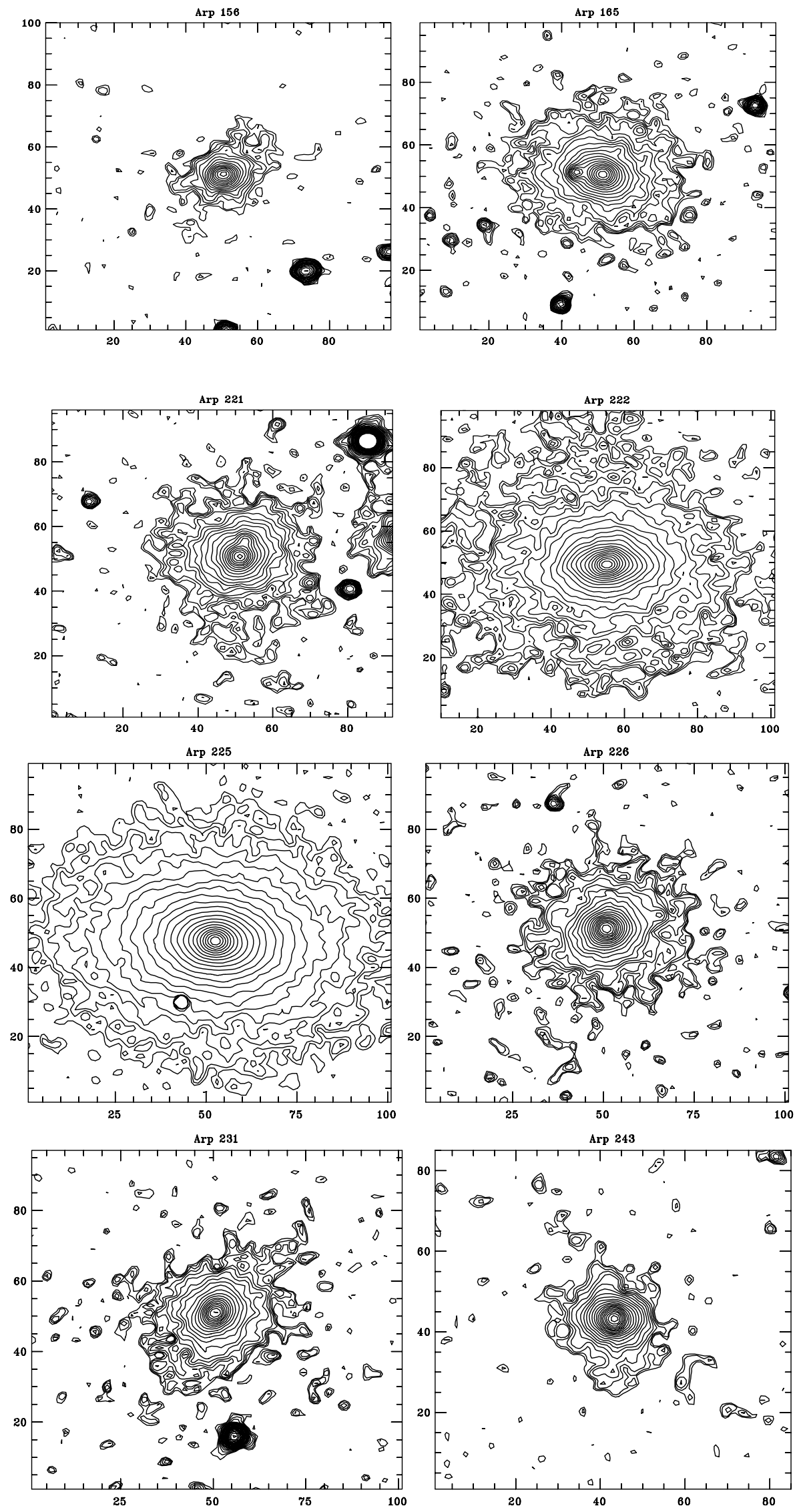

Fig. 1. $K_{\mathrm{s}}$ band contours of the sample galaxies. The contours are plotted at intervals of $0.25^{\mathrm{m}}$ with the faintest contour level being $19^{\mathrm{m}}$ for all the galaxies except for Arp 193, Arp 221, Arp 231 and Arp 243 for which the faintest contour is at $19.5^{\mathrm{m}}$ and at $18.5^{\mathrm{m}}$ for Arp 225. The $X$ and $Y$ axes are given in arcsecs. 

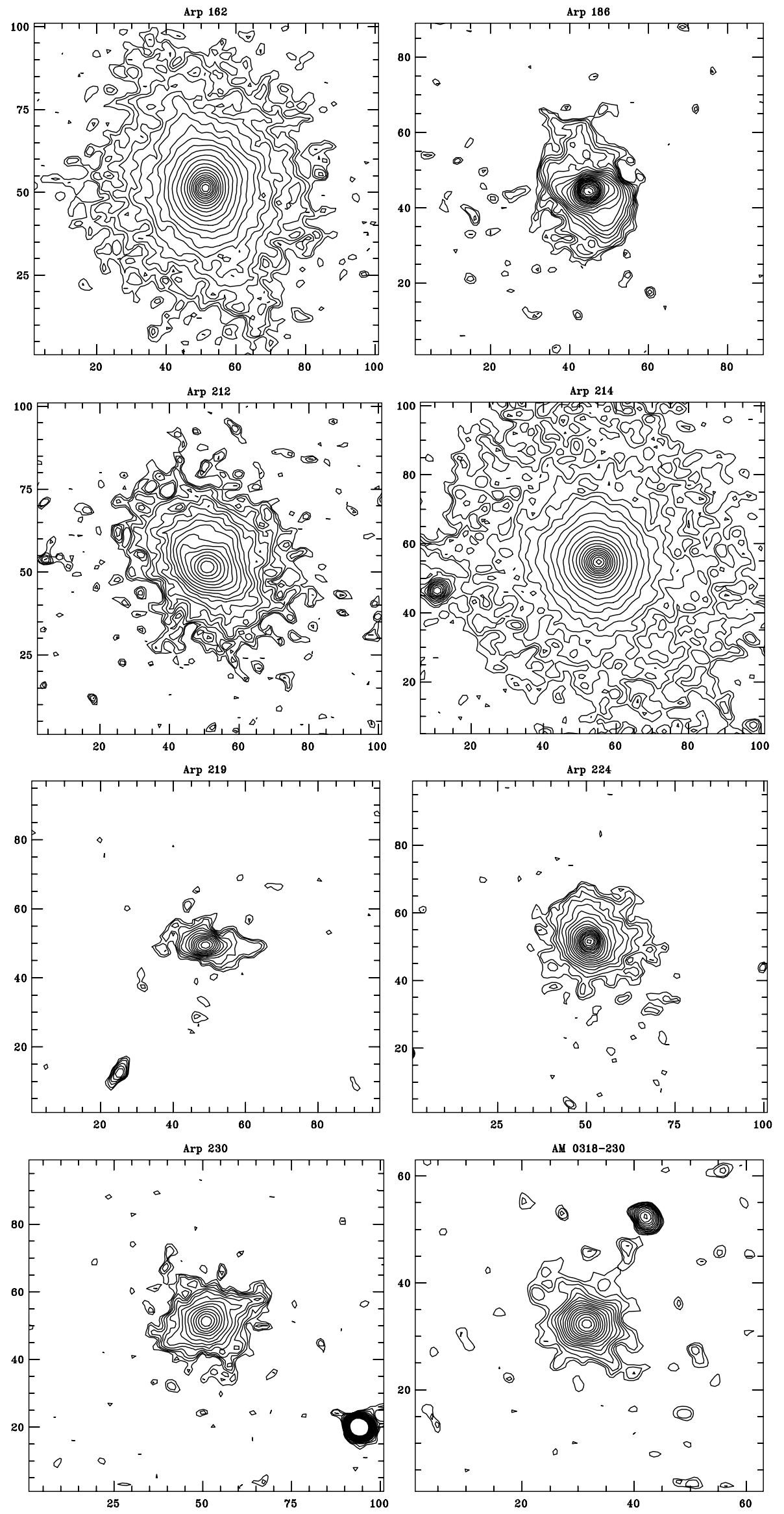

Fig. 1. continued. 

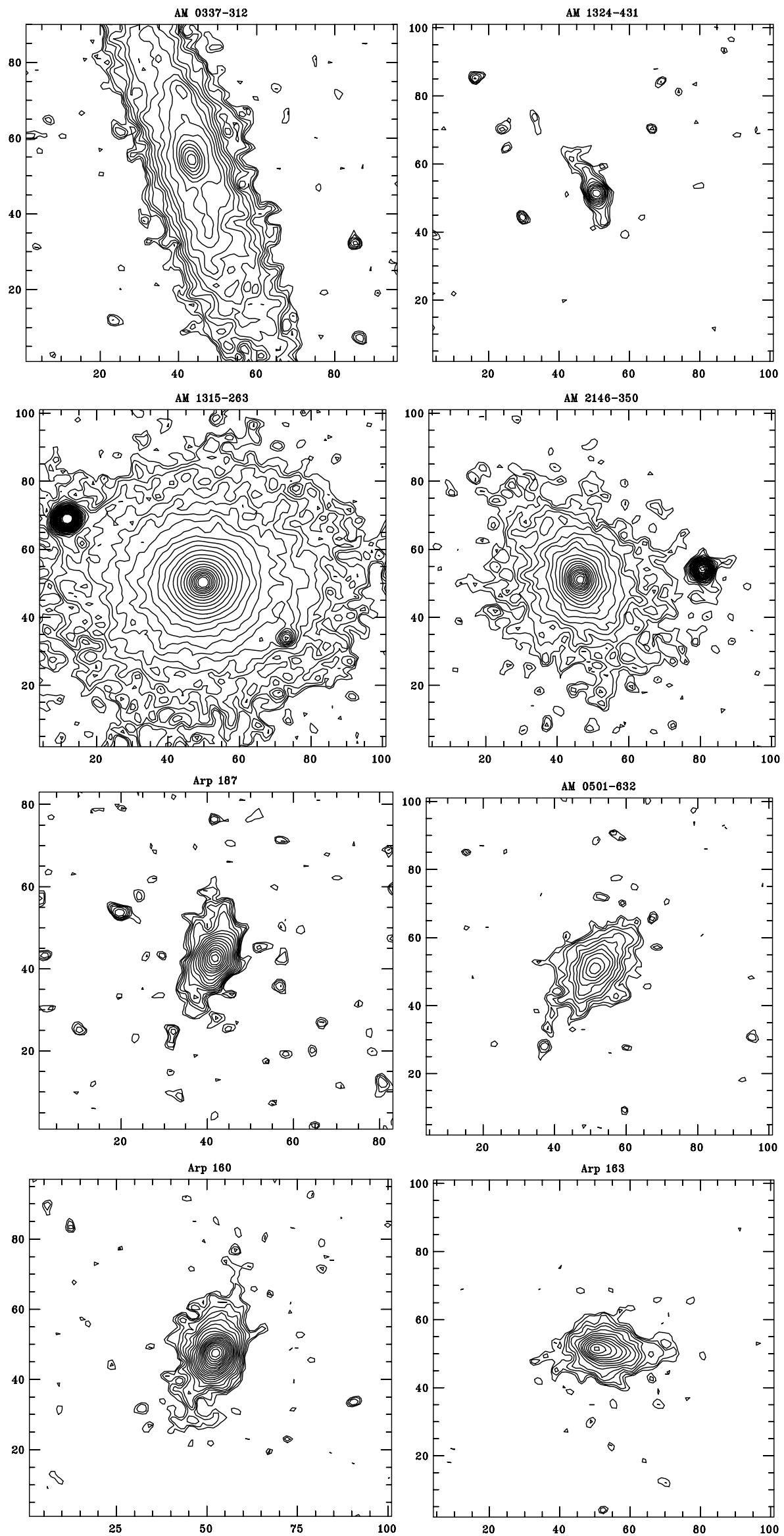

Fig. 1. continued. 

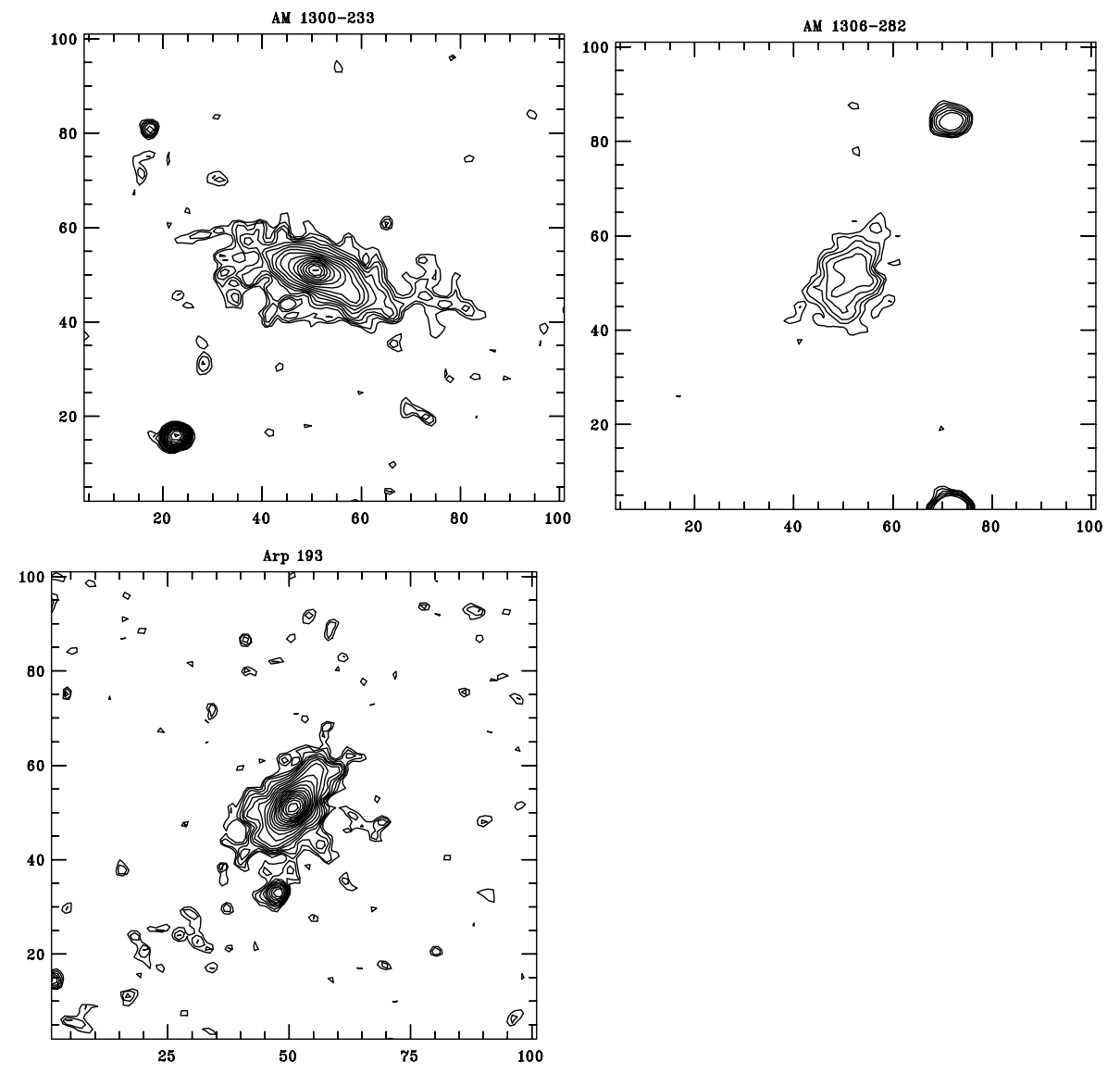

Fig. 1. continued.

The first step was to fit the surface brightness profiles using de Vaucouleur's law. The fits were made using the task nfit1d within STSDAS. The fit was tried between the radius of $2^{\prime \prime}$ to the radius where the signal falls to $1 \sigma$ of the background noise level. It was found that 9 of the 27 sample objects could be fit by an $r^{1 / 4}$ law over this entire range. The surface brightness profiles and the fits for these 9 galaxies are shown in Fig. 2. The profiles of 13 of the remaining galaxies clearly show the presence of an outer exponential disk. We fit this outer exponential and the fits are shown in Fig. 3. The error bars in both these figures are small except in the outer regions in some cases. The resulting scale lengths for the two classes are given in Table 3. The columns in Table 3 are: Col. 1: the name of the galaxy; Col. 2: the distance to the galaxy in $\mathrm{Mpc}$; Col. 3: the linear resolution of the galaxy in pc per arcsec; Col. 4: the scale length (the bulge scale length in class I and the disk scale length in class II) in kpc; Col. 5: the surface brightness; Col. 6: the concentration index $C_{31}$; Col. 7: the central velocity dispersion from HYPERCAT (http://www-obs.univ-lyon1.fr/hypercat/) and Col. 8: the spectral type for the central region taken from SIMBAD and ADS. Of the remaining 5 galaxies, one (AM 0501-632) could be fit by a single exponential throughout, while the other 4 (Arp 160, Arp 163, AM 1300-233 and AM 1306-282) could not be fit by either law. These galaxies show a highly disturbed surface brightness profile (Fig. 4).

\subsection{Luminosity profile classes}

Based on the behaviour of the surface brightness profiles, we classify the sample objects into two main classes:

Class I: the surface brightness profile of these galaxies is well fit by an $r^{1 / 4}$ law throughout most of its range. The fits to Arp 165, Arp 221, Arp 222 and Arp 225 are very good right up to the outermost data point, as shown in Fig. 2. These systems have presumably relaxed completely, resulting in ellipse-like profiles. A few cases, like Arp 156 , Arp 193, Arp 226, Arp 231 and Arp 243, show slight deviations in the outermost regions, which may not have relaxed completely. Alternatively, the small deviations in the outermost regions could just be the typical behaviour shown by normal ellipticals at larger radii (Binney \& Merrifield 1998).

Class II: the surface brightness profiles of this class of galaxy could not be fit solely by a single $r^{1 / 4}$. When the fit was tried, we found that there was an excess over the fit in the outer regions. A bulge plus disk also was tried and it does not give a good fit. In the case of Arp 212 and AM 0318-230, even the inner region could not be fit by an $r^{1 / 4}$ law. In general, we found that the combined fit was highly sensitive to the range over which the fit was made. All these galaxies are best fit by an exponential disk in the outer parts as shown in Fig. 3. It should be noted that this exponential typically fits very well over more than 2 scale lengths. 
Table 1. The sample.

\begin{tabular}{|c|c|c|c|c|c|}
\hline Galaxy & Alt.name & $\mathrm{RA}(2000)$ & $\operatorname{Dec}(2000)$ & Type(RC3) & Type(SIMBAD) \\
\hline Arp 156 & UGC 5814 & $10: 42: 38.0$ & $+77: 29: 41$ & - & - \\
\hline Arp 160 & NGC 4194 & $12: 14: 09.8$ & $+54: 31: 39$ & IBm pec & I \\
\hline Arp 162 & NGC 3414 & $0: 51: 16.3$ & $+27: 58: 33$ & S0 pec & SB0 \\
\hline Arp 163 & NGC 4670 & $12: 45: 17.0$ & $+27: 07: 34$ & $\mathrm{SB}(\mathrm{s}) 0 / \mathrm{a} \mathrm{pec}$ & S0 \\
\hline Arp 165 & NGC 2418 & $07: 36: 37.9$ & $+17: 53: 06$ & $\mathrm{E}$ & $\mathrm{E}$ \\
\hline Arp 186 & NGC 1614 & $04: 33: 59.9$ & $-08: 34: 30$ & $\mathrm{SB}(\mathrm{s}) \mathrm{c}$ pec & I \\
\hline Arp 187 & - & 05:04:52.99 & $-10: 14: 51$ & - & - \\
\hline Arp 193 & IC 883 & $13: 20: 35.3$ & $+34: 08: 22$ & Im: pec & - \\
\hline Arp 212 & NGC 7625 & $23: 20: 30.8$ & $+17: 13: 41$ & $\mathrm{SA}(\mathrm{rs}) \mathrm{a}$ pec & Sp \\
\hline Arp 214 & NGC 3718 & $11: 32: 35.7$ & $+53: 03: 59$ & SB(s)a pec & $\mathrm{SB} 0 / \mathrm{Sa}$ \\
\hline Arp 219 & - & 03:39:53.26 & $-02: 06: 47$ & - & $\mathrm{SB}+\mathrm{cG}$ \\
\hline Arp 221 & - & 09:36:28.03 & $-11: 19: 49$ & - & - \\
\hline Arp 222 & NGC 7727 & 23:39:53.8 & $-12: 17: 36$ & SAB(s)a pec & $\mathrm{Sa}$ \\
\hline Arp 224 & NGC 3921 & 11:51:06.1 & $+55: 04: 39$ & $\left(\mathrm{R}^{\prime}\right) \mathrm{SA}(\mathrm{s}) 0 /$ a pec & S0 \\
\hline Arp 225 & NGC 2655 & $08: 55: 38.7$ & $+78: 13: 28$ & $\mathrm{SAB}(\mathrm{s}) 0 / \mathrm{a}$ & $\mathrm{S} 0 / \mathrm{Sa}$ \\
\hline Arp 226 & NGC 7252 & $22: 20: 44.9$ & $-24: 40: 41$ & $(\mathrm{R}) \mathrm{SA}(\mathrm{r})$ & - \\
\hline Arp 230 & IC 51 & $00: 46: 24.3$ & $-13: 26: 34$ & S0 pec? & - \\
\hline Arp 231 & PGC 2616 & $00: 43: 54.3$ & $-04: 14: 43$ & - & $\mathrm{S} 0+$ \\
\hline Arp 243 & NGC 2623 & $08: 38: 24.2$ & $+25: 45: 01$ & - & - \\
\hline AM 0318-230 & PGC 12526 & 03:20:40.1 & $-22: 55: 50$ & $\mathrm{~S} ?$ & $\mathrm{Sa}$ \\
\hline AM 0337-312 & NGC 1406 & $03: 39: 22.6$ & $-31: 19: 19$ & SB(s)bc: sp & $\mathrm{Sc}$ \\
\hline AM 1315-263 & NGC 5061 & $13: 18: 04.9$ & $-26: 50: 10$ & E0 & $\mathrm{E}$ \\
\hline AM 2146-350 & NGC 7135 & $21: 49: 43.7$ & $-34: 52: 42$ & SA0- pec & $\mathrm{S}$ \\
\hline AM 0501-632 & PGC 16567 & 05:01:30.1 & $-63: 17: 34$ & $\mathrm{~S} 0+?$ pec & $\mathrm{S}$ \\
\hline AM 1300-233 & MGC-4-31-23 & 13:02:52.07 & $-23: 55: 19$ & - & Ir \\
\hline AM 1306-282 & PGC 45596 & 13:09:10.4 & $-28: 38: 23$ & $\mathrm{SAB}(\mathrm{rs}) \mathrm{s}$ pec? & $\mathrm{Sa}$ \\
\hline AM 1324-431 & PGC 47188 & $13: 27: 51.0$ & $-43: 25: 50$ & $\mathrm{~S} ?$ & $\mathrm{Sc}$ \\
\hline
\end{tabular}

Four of the sample galaxies could not be fitted by an $r^{1 / 4}$ or an exponential profile. These systems may not yet have fully relaxed, as suggested by their structural parameters, like the wandering centre (See Sect. 3.3). The surface brightness profiles of these galaxies are shown in Fig. 4.

Of the 27 galaxies in our sample, 9 belong to Class I, 13 belong to Class II and 4 do not belong to either class. The galaxies Arp 222 and Arp 225 from Class I have radii greater than $50^{\prime \prime}$ and the possible presence of an outer exponential disk beyond $50^{\prime \prime}$ cannot be ruled out completely. However, the $r^{1 / 4}$ law gives a good fit up to the outermost point as is seen in Fig. 3. We cannot make a clear statistical remark about the percentages belonging to each class since our sample is not a complete one.

Information on the nature of the luminosity profiles of some of our sample galaxies exists in the literature and a brief description is given below: Arp 162, Arp 214, AM 0337-312 and Arp 163 show an inner truncated exponential disk in the photographic $V$ band and Arp 225 shows only an $r^{1 / 4}$ profile (Baggett et al. 1998). Arp 212 shows an outer exponential in the $B$ band ( $\mathrm{Lu} \mathrm{1998).}$ Scoville et al. (2000) find that the brightness profile of Arp 243 in the $H$ and $K$ bands can be approximated by an $r^{1 / 4}$ profile and the $H$ band profile of Arp 193 is better fit by an $r^{1 / 4}$ law than an exponential law, based on
HST data. For Arp 224, we find that the outer part can be fit quite well by an exponential profile as is clear from the small error bars in Fig. 3. SB91 report that the $K$ band surface brightness profile can be fit by an $r^{1 / 4}$ law only in the inner $8^{\prime \prime}$ and the profile deviates from an $r^{1 / 4}$ law beyond this point. Schweizer (1996) finds no evidence for the presence of an outer exponential disk in the $R$ band images of Arp 224.

\subsection{Structural parameters}

In addition to the luminosity profile, the results of the isophotal analysis also provide valuable information about other structural and the dynamical parameters of the galaxies. The coefficient $(B 4)$ of the $\cos (4 \theta)$ component is a measure of the diskiness (if $B 4>0$ ) or boxiness (if $B 4<0$ ) of the galaxy. Studies of elliptical galaxies show that boxiness is an indicator of a merger as the origin of some ellipticals (Nieto \& Bender 1989). Our sample however shows a disturbed morphology, yet is fit by an $r^{1 / 4}$ law (Class I). Therefore, it is not clear what physical significance the values of $B 4$ have for these disturbed systems. Nevertheless, we give these trends in Appendix A for each galaxy, which may be used as input for future theory work by others. The analysis also provides information about the shift in the centre for successive isophotes. 
Table 2. Basic data.

\begin{tabular}{|c|c|c|c|c|c|}
\hline Galaxy & $f_{100} / f_{60}$ & $\begin{array}{l}L_{\mathrm{IR}} \\
\left(\times 10^{10} L_{\odot}\right)\end{array}$ & $M_{B}$ & $\begin{array}{l}\text { radius }^{\prime \prime} \\
\text { 2MASS }\end{array}$ & $\begin{array}{l}K_{\mathrm{s}} \\
2 \mathrm{MASS}\end{array}$ \\
\hline Arp 156 & 2.61 & $<0.21$ & -17.142 & 26.8 & 10.534 \\
\hline Arp 160 & 1.21 & 7.62 & -20.322 & 25.6 & 9.680 \\
\hline Arp 162 & $<4.8$ & $<0.07$ & -19.606 & 64.4 & 8.059 \\
\hline Arp 163 & 1.69 & $<0.21$ & -17.9 & 30 & 10.612 \\
\hline Arp 165 & - & $<0.27$ & -20.76 & 40.6 & 9.037 \\
\hline Arp 186 & 1.01 & 39.4 & -21.193 & 24.2 & 9.565 \\
\hline Arp 187 & - & - & - & 16.8 & 11.314 \\
\hline Arp 193 & 1.63 & 36.9 & -20.38 & 19.6 & 10.895 \\
\hline Arp 212 & 1.90 & 1.45 & -19.081 & 42.8 & 8.929 \\
\hline Arp 214 & 3.28 & 0.07 & -19.818 & 80 & 7.852 \\
\hline Arp 219 & 2.02 & $<5.69$ & -21.411 & 19.2 & 11.462 \\
\hline Arp 221 & 1.63 & - & -20.376 & 33.8 & 9.491 \\
\hline Arp 222 & - & - & -20.546 & 68.6 & 7.797 \\
\hline Arp 224 & $<2.45$ & $<2.42$ & -21.58 & 27.8 & 9.939 \\
\hline Arp 225 & 2.98 & 0.25 & -20.803 & 80 & 7.223 \\
\hline Arp 226 & 1.76 & 5.31 & -20.926 & 33.2 & 9.399 \\
\hline Arp 230 & 2.12 & 0.41 & -18.089 & 25.6 & 10.364 \\
\hline Arp 231 & - & - & -19.713 & 26.4 & 9.887 \\
\hline Arp 243 & 1.20 & 29.33 & -21.205 & 19.8 & 10.505 \\
\hline AM 0318-230 & - & - & -20.568 & 17.2 & 11.166 \\
\hline AM 0337-312 & 2.26 & 0.82 & -18.506 & 80 & 8.823 \\
\hline AM 1315-263 & - & $<0.07$ & -21.118 & 71.8 & 7.372 \\
\hline AM 2146-350 & 3.5 & $<0.20$ & -19.579 & 51 & 8.998 \\
\hline AM 0501-632 & 2.60 & 0.06 & -17.111 & 24.2 & 10.851 \\
\hline AM $1300-233$ & 1.11 & 23.61 & -21.282 & 37.4 & 10.281 \\
\hline AM 1306-282 & 2.05 & $<0.12$ & -18.372 & 23.4 & 11.291 \\
\hline AM 1324-431 & 1.31 & $<10.74$ & -21.293 & 18 & 12.239 \\
\hline
\end{tabular}

The $x-y$ coordinates of the centre of each fitted isophote can tell us whether the isophote is concentric or otherwise. If the isophotes are not concentric, it means that there is asymmetry in the central mass distribution.

Details on each object can be found in Appendix A. Of the 27 sample galaxies, we find that 10 show only diskiness, 4 show only boxiness while 8 show both boxiness and diskiness. 5 galaxies do not show any clear trend towards diskiness or boxiness. For the Classes I and II, we find that the inner isophotes are concentric. The coordinates of the centre start changing for the outer isophotes. In contrast, for all the unrelaxed objects shown in Fig. 4, the isophotes are non-concentric right from the innermost regions. That is, the centres of the isophotes show a wandering or a "sloshing" pattern, indicating the dynamically unrelaxed behaviour of the merger even in the central regions. This difference is clearly illustrated in Fig. 5 where the quantities derived from the isophotal analysis, namely $A 4, B 4, X_{0}$ and $Y_{0}$ are presented for one galaxy belonging to Class I (Arp 193) and one which could not be fit by an $r^{1 / 4}$ or an exponential disk (Arp 163).

\subsection{Concentration index}

The scale lengths derived from ellipse fitting depend on the structure namely, the ellipticity and the position angle of the fitted isophotes. In order to compare the scale lengths etc. from morphologically dissimilar galaxies, it is more accurate to use an index that does not change with the morphology. To have a model-independent handle on the concentration of the light in the galaxy, we derive the ratio of the radius that encloses $75 \%$ of the total light to the radius that encloses $25 \%$ of the total light. The total light was the light enclosed within an elliptical isophote at the $20 \mathrm{mag} \operatorname{arcsec}^{-2}$ level and was taken from the 2MASS database. This ratio is called the concentration index $C_{31}$ (de Vaucouleurs 1977) and is presented in Table 3. Plotting the frequency distribution of $C_{31}$ in the two classes (Fig. 6), we find that Class I objects show a peak at a value between 4 and 5 while Class II objects show a double peaked distribution with a gap in between. The gap corresponds to the region occupied by Class I objects. We find that the highest $C_{31}$ objects are made up 
Table 3. Properties of the sample galaxies.

\begin{tabular}{|c|c|c|c|c|c|c|c|}
\hline Galaxy & $\begin{array}{c}D \\
(\mathrm{Mpc})\end{array}$ & $\begin{array}{l}1^{\prime \prime} \\
(\mathrm{pc})\end{array}$ & $\begin{array}{c}R_{\mathrm{e}}, R_{\mathrm{d}} \\
(\mathrm{kpc})\end{array}$ & $\begin{array}{c}\mu \\
\left(\mathrm{mag}_{\operatorname{arcsec}}{ }^{-2}\right)\end{array}$ & $C_{31}$ & $\begin{array}{c}\sigma \\
\left(\mathrm{km} \mathrm{s}^{-1}\right)\end{array}$ & Spectral type \\
\hline Class I & & & $R_{\mathrm{e}}$ & $\mu_{\mathrm{e}}$ & & & \\
\hline Arp 156 & 25 & 121 & $1.03 \pm 0.14$ & 18.08 & 4.35 & - & - \\
\hline Arp 165 & 67 & 325 & $5.92 \pm 0.76$ & 18.37 & 4.98 & 268 & -early type \\
\hline Arp 193 & 93 & 451 & $3.89 \pm 0.38$ & 17.89 & 3.68 & 206 & LINER, starburst \\
\hline Arp 221 & - & - & - & 18.32 & 4.18 & - & - \\
\hline Arp 222 & 25 & 125 & $2.83 \pm 0.46$ & 17.43 & 5.23 & 200 & - \\
\hline Arp 225 & 18 & 91 & $3.26 \pm 0.38$ & 17.70 & 4.86 & 164 & LINER,early type \\
\hline Arp 226 & 64 & 312 & $3.28 \pm 0.77$ & 17.43 & 4.74 & 177 & - \\
\hline Arp 231 & 76 & 368 & $3.80 \pm 0.45$ & 17.77 & 4.13 & - & - \\
\hline Arp 243 & 72 & 351 & $1.58 \pm 0.36$ & 16.68 & 4.07 & 95 & LINER, starburst \\
\hline Class II & & & $R_{\mathrm{d}}$ & $\mu_{0}$ & & & \\
\hline Arp 162 & 17 & 87 & $1.56 \pm 0.04$ & 16.37 & 5.50 & 250 & LINER \\
\hline Arp 186 & 63 & 308 & $1.69 \pm 0.09$ & 15.33 & 4.62 & $150 ?$ & AGN \\
\hline Arp 187 & 164 & 795 & $4.05 \pm 0.19$ & 16.44 & 3.24 & - & - \\
\hline Arp 212 & 21 & 104 & $0.85 \pm 0.03$ & 15.26 & 3.33 & - & starburst \\
\hline Arp 214 & 13 & 65 & $1.72 \pm 0.11$ & 16.96 & 5.41 & 178 & LINER \\
\hline Arp 219 & 139 & 673 & $4.86 \pm 0.22$ & 17.01 & 3.47 & - & - \\
\hline Arp 224 & 79 & 383 & $2.91 \pm 0.21$ & 16.57 & 5.14 & 195 & post starburst \\
\hline Arp 230 & 23 & 113 & $0.75 \pm 0.02$ & 16.21 & 3.17 & - & - \\
\hline AM 0318-230 & 142 & 692 & $4.76 \pm 0.51$ & 17.34 & 3.88 & - & - \\
\hline AM 0337-312 & 14 & 69 & $2.28 \pm 0.07$ & 16.21 & 3.33 & - & - \\
\hline AM 1324-431 & 139 & 675 & $4.39 \pm 0.34$ & 17.60 & 3.83 & - & - \\
\hline AM 1315-263 & 24 & 116 & $1.90 \pm 0.04$ & 15.65 & 5.66 & 194 & - \\
\hline AM 2146-350 & 36 & 174 & $3.22 \pm 0.23$ & 17.17 & 5.22 & - & LINER \\
\hline \multicolumn{8}{|l|}{ No fit } \\
\hline AM 0501-632 & 14 & 69 & $0.41 \pm 0.003$ & 16.19 & 2.69 & - & - \\
\hline Arp 160 & 34 & 168 & - & - & 3.21 & 104 & starburst,AGN \\
\hline Arp 163 & 16 & 78 & - & - & 3.52 & - & Wolf Rayet, HII \\
\hline AM 1300-233 & 86 & 417 & - & - & 4.47 & - & Seyfert \\
\hline AM 1306-282 & 19 & 92 & - & - & 2.63 & - & - \\
\hline
\end{tabular}

largely of galaxies with a nuclear AGN/LINER component (except for Arp 224 and AM 1315-263), as shown by the shading. The left peak corresponding to a lower $C_{31}$ is due to non-active objects. Thus, active galaxies having an outer exponential profile are found to be more centrally concentrated as compared to the $r^{1 / 4}$ (completely relaxed) systems which in turn are more centrally concentrated than non-active galaxies having an outer exponential profile. Apparently, the AGN in the central region of the Class II galaxies contributes to a great extent to the total light in the centre, as a result of which they appear more centrally concentrated compared to the non-active Class II galaxies.

The fitted values of the bulge $\left(R_{\mathrm{e}}\right)$ and disk $\left(R_{\mathrm{d}}\right)$ scale lengths and the surface brightnesses $\left(\mu_{\mathrm{e}}\right.$ and $\mu_{0}$ for Class I and Class II respectively) for the sample galaxies obtained in Sect. 3.1 are given in Table 3. We used the velocity measurements from SIMBAD to derive the distances to the galaxies and to compute the linear resolutions. A value of $H_{0}=75 \mathrm{~km} \mathrm{~s}^{-1} \mathrm{Mpc}^{-1}$ is used throughout. The derived quantities are listed in Table 3.

\section{Results}

\subsection{Dynamical properties}

Despite their similar optical appearance and hence a similar stage of dynamical evolution of tidal features, the galaxies seem to fall into two distinct groups with different mass profiles. Thus, the galaxies in the two classes have undergone a very different evolutionary pattern. The natural physical explanation of the Class I galaxies is that each is a largely relaxed merger of equal mass giant galaxies as studied in typical numerical simulations 

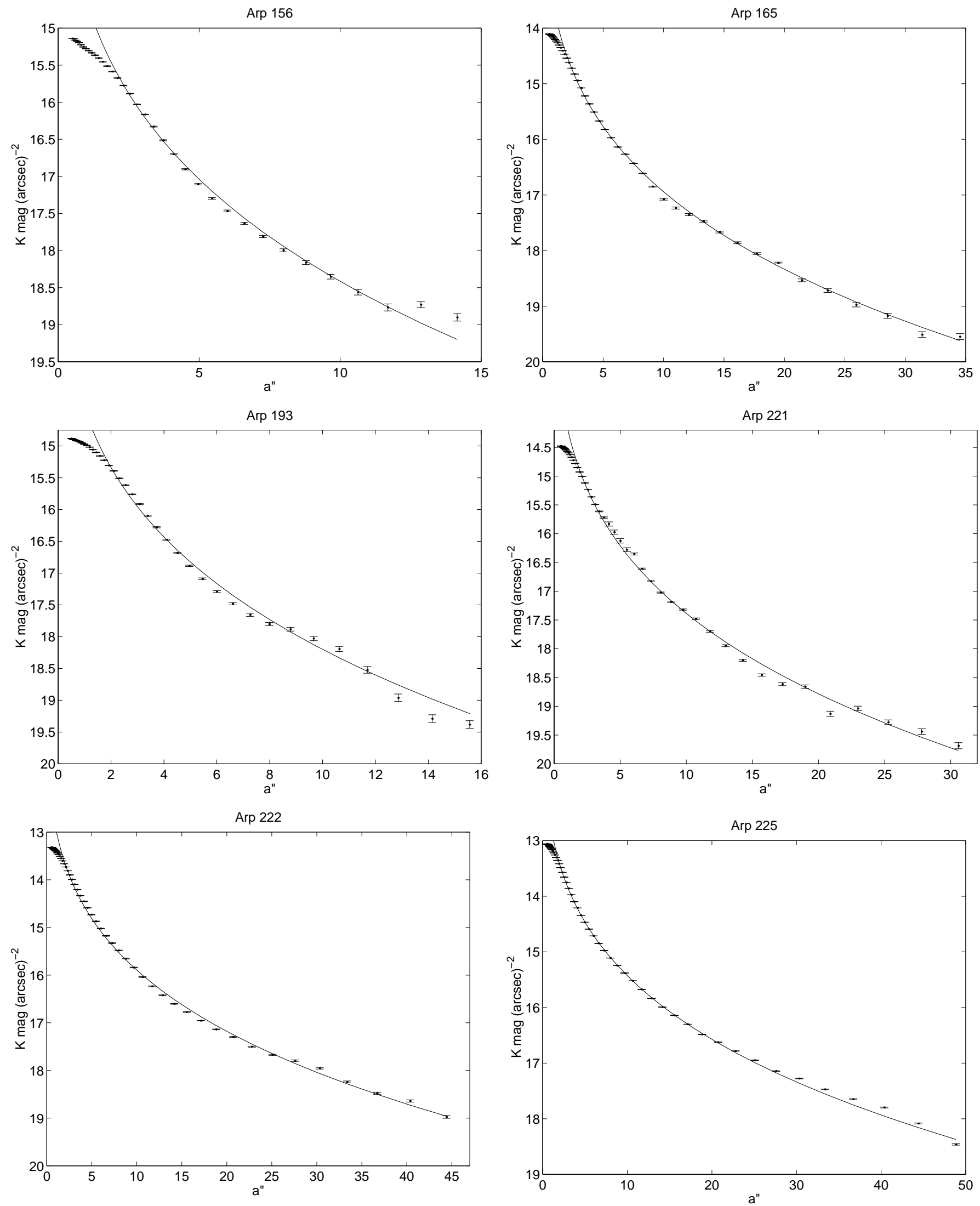

Fig. 2. Class I: galaxies well fit by an $r^{1 / 4}$ law. The $K_{\mathrm{s}}$ band magnitude in mag $\operatorname{arcsec}^{-2}$ is plotted against the semi-major axis, $a^{\prime \prime}$. 

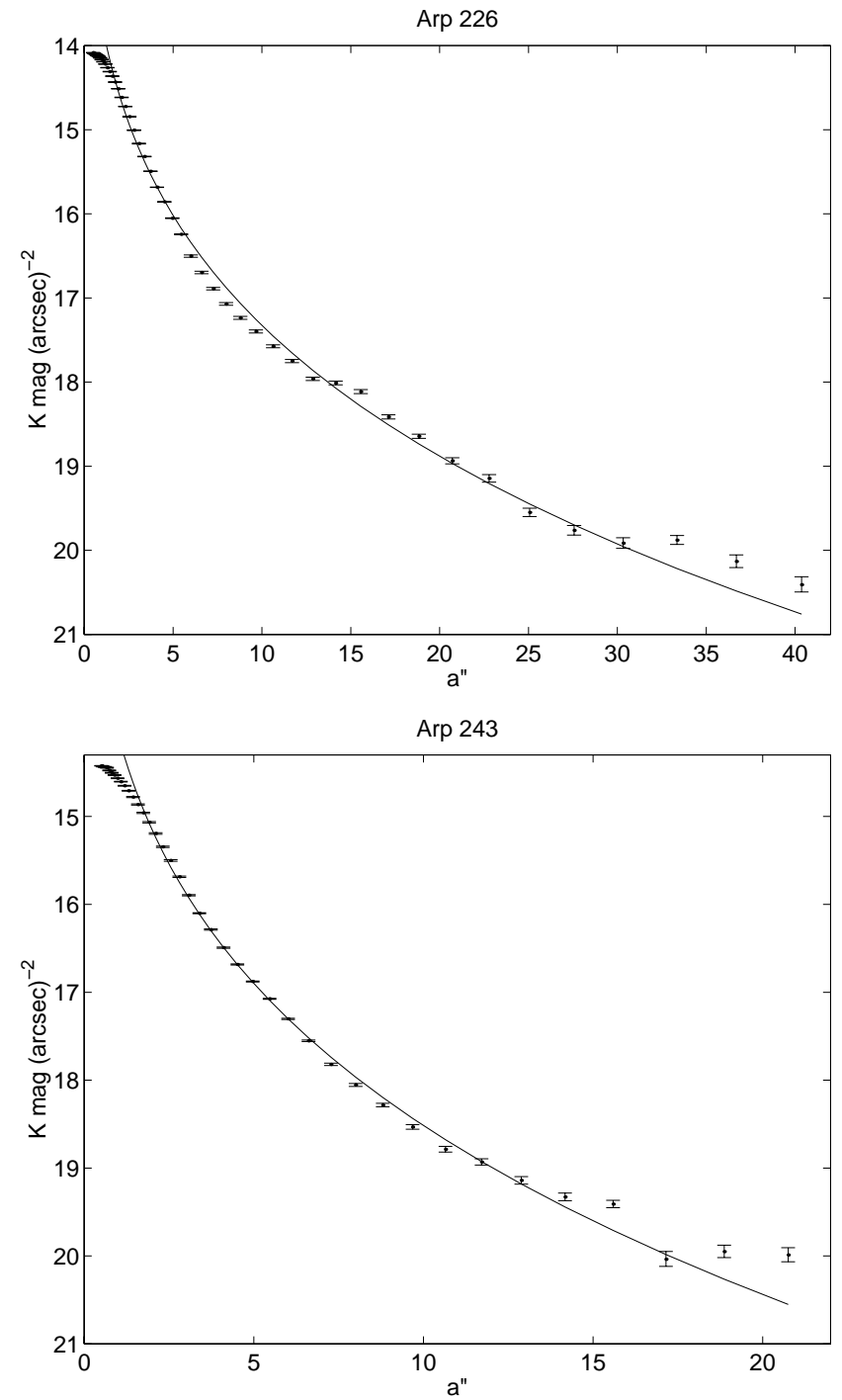

Fig. 2. continued.

(e.g. Barnes \& Hernquist 1991). The origin of the Class II galaxies is a puzzle. It may be that the galaxies in Class II undergo a merger with a satellite or a minor merger with a mass ratio $\geq 0.1$ and hence the exponential disk in the larger galaxy is not significantly disturbed. This conjecture for Class II is supported by the observations of NGC 4424 by Kenney et al. (1996) who report that morphological features generally associated with a merger are seen in this case and the outer part of the galaxy has a disk-like exponential light profile. They argue that the apparent survival of the dominant exponential disk in this galaxy suggests that it has experienced an intermediate mass ratio (0.1-0.5) merger. Further, Bekki (1998b) proposes unequal mass mergers with ratios $(0.3-0.5)$ as the mechanism for the creation of S0 galaxies with outer exponential disks (also see Barnes 1998). Thus, we propose that $K_{\mathrm{s}}$ band photometry, in particular the radial profile, can be used as a diagnostic to differentiate between cases of equal-mass and unequal-mass merger remnants and to place a limit on the possible mass ratios in an unequalmass merger.

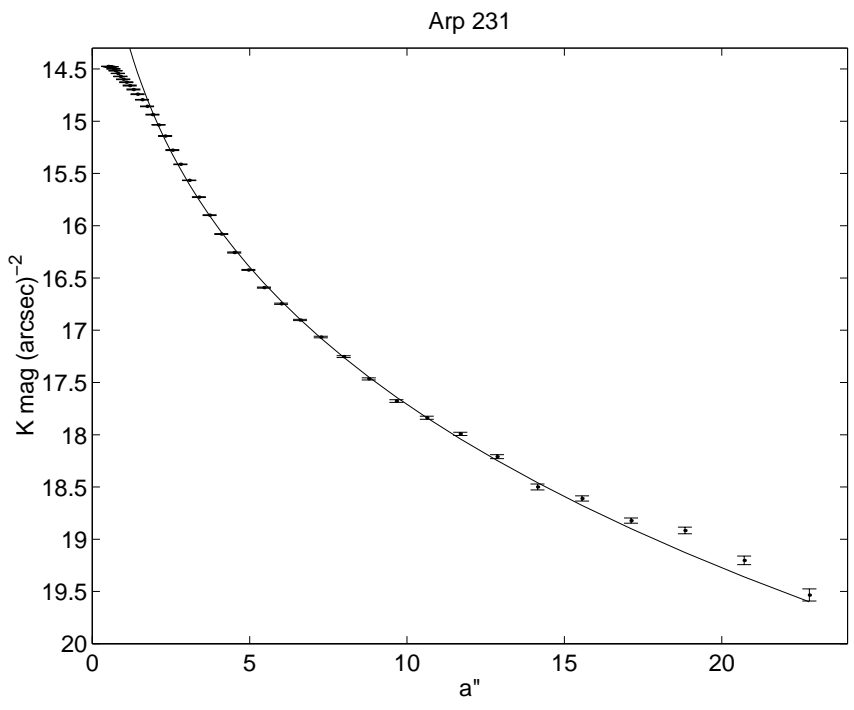

Our scenario for the origin of Class II galaxies is supported by numerical simulations of minor mergers by Walker et al. (1996). They show that the final stage of a minor merger still resembles a disk galaxy but has a thicker and hotter disk. The remnants older than $6 \times$ $10^{8}$ years (see their Fig. 5) appear remarkably similar to the optical appearance of the sample we have chosen in our study. However, these authors do not give the resulting radial mass distribution. Our work shows that the exponential mass distribution in the outer disks, as seen from the large range in radius over which it fits (Sect. 3.2) and the fact that the error bars are small (Fig. 3), shows that the exponential nature is robust for the Class II galaxies.

We note that the theoretical work so far has mostly concentrated on studying a merger of equal mass galaxies (see Sect. 1), because the focus has been mainly to prove that a merger of spirals can give rise to an elliptical galaxy, though the unequal-mass encounters are also now being studied (e.g. Barnes 1998). The dynamical evolution of merging galaxies resulting in an exponential outer profile as observed in Class II is an open problem, and 

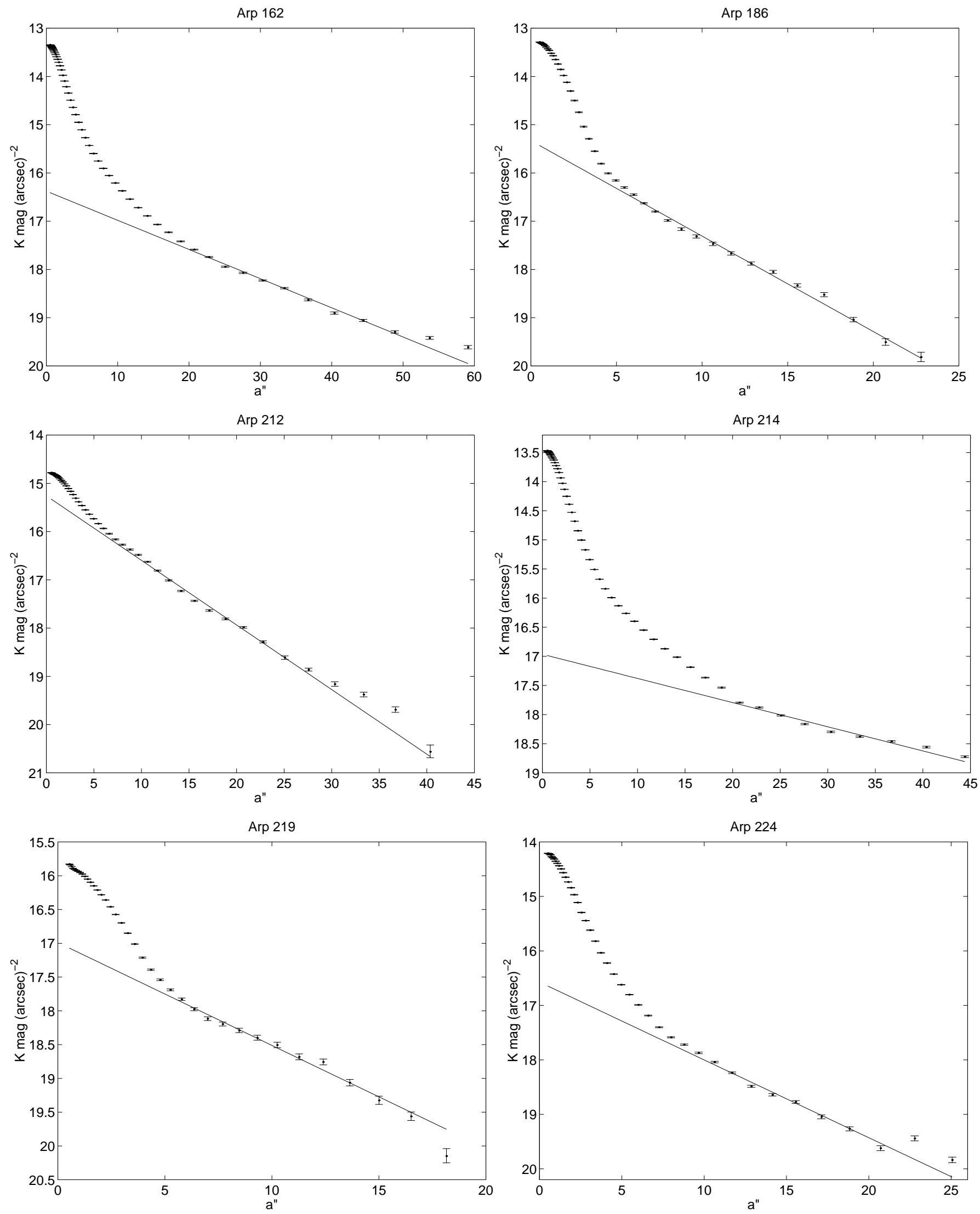

Fig. 3. Class II: galaxies well fit by an outer exponential disk. The $K_{\mathrm{s}}$ band magnitude in mag arcsec ${ }^{-2}$ is plotted against the semi-major axis, $a^{\prime \prime}$. 

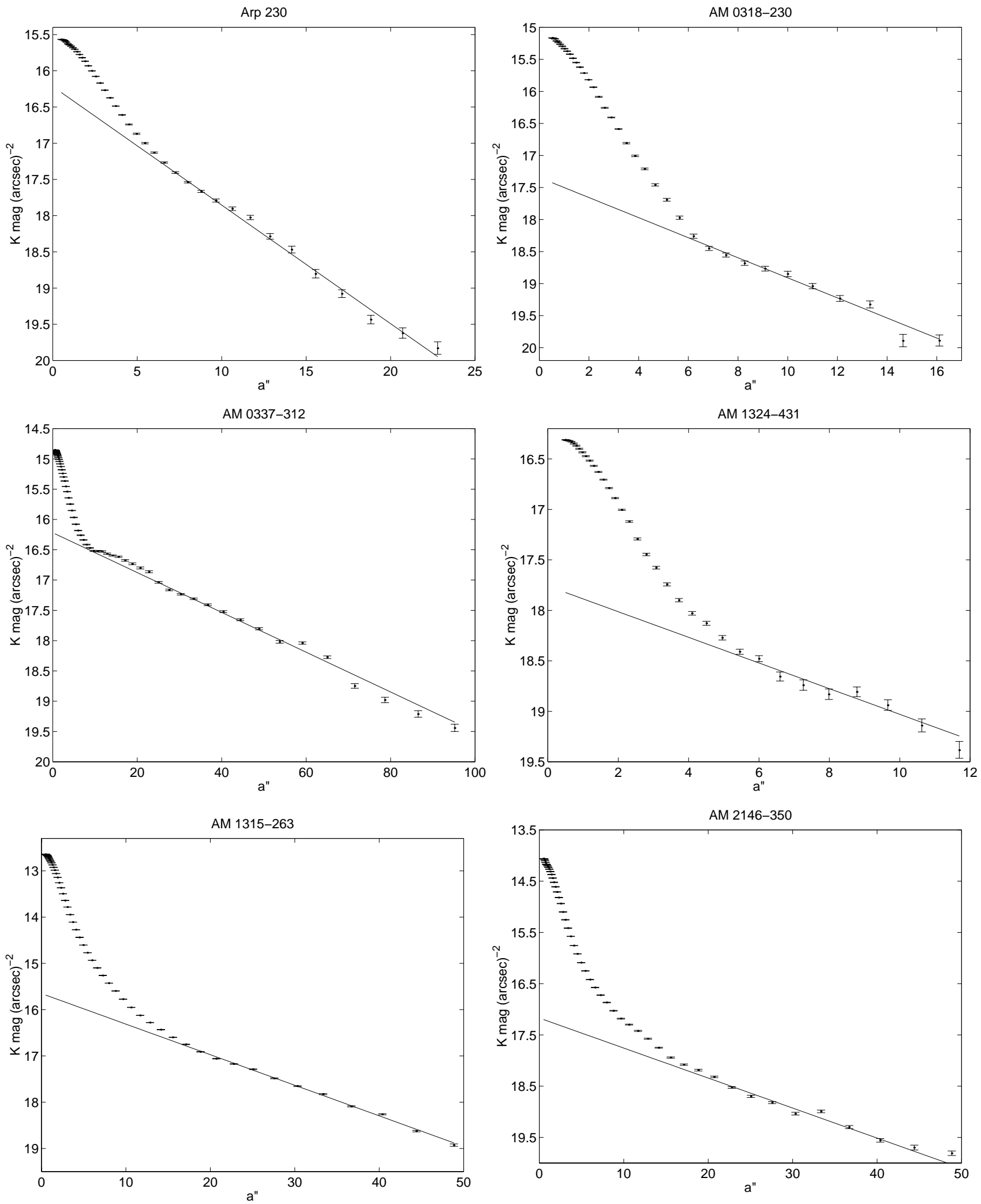

Fig. 3. continued.

needs to be further studied by future $N$-body work. It is puzzling that the second oldest remnant in the Toomre sequence, namely Arp 224, should show an exponential disk behaviour in the outer region. It is difficult to explain how a merging pair can have a disturbed 3-D light

distribution and yet have a smooth, exponential disk mass distribution, as in a typical spiral galaxy.

Recently, Naab \& Burkert (2001) have performed $N$-body simulations of merging disk galaxies with mass ratios of $1: 1,2: 1,3: 1$ and $4: 1$. They predict the kinematic 

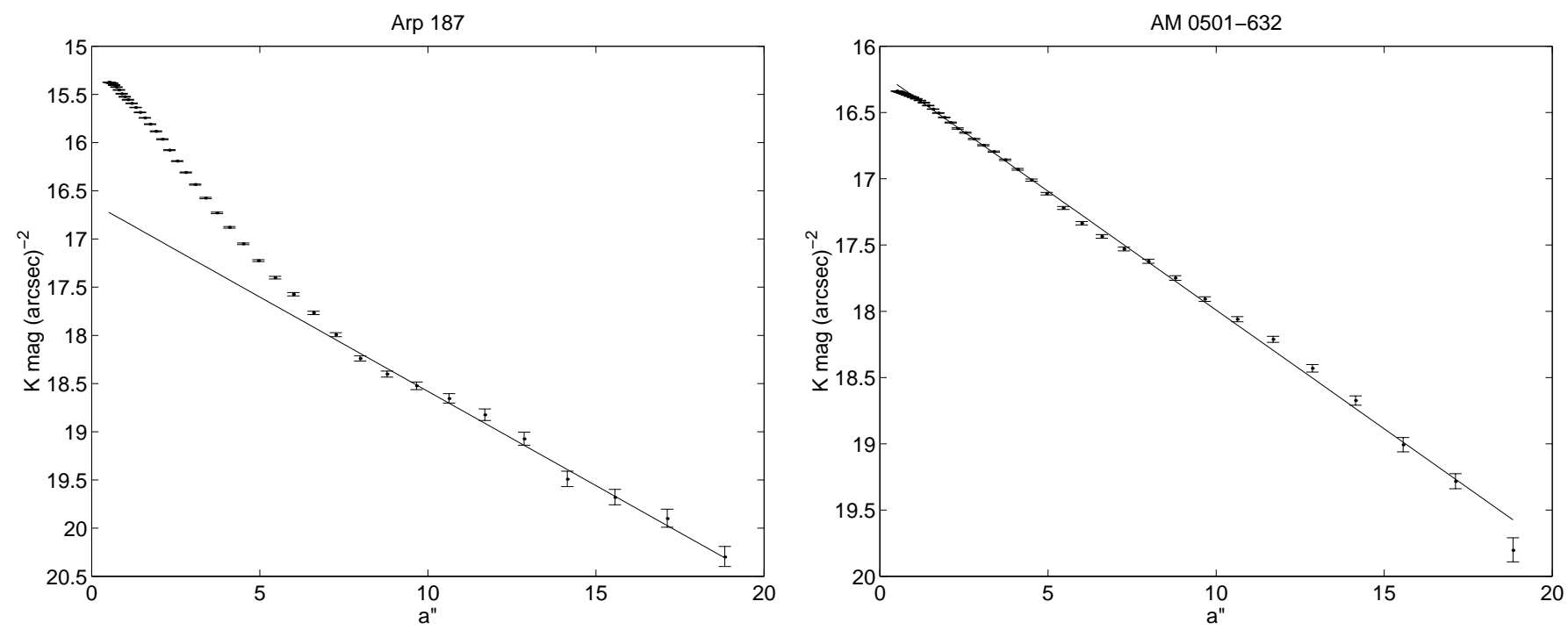

Fig. 3. continued.
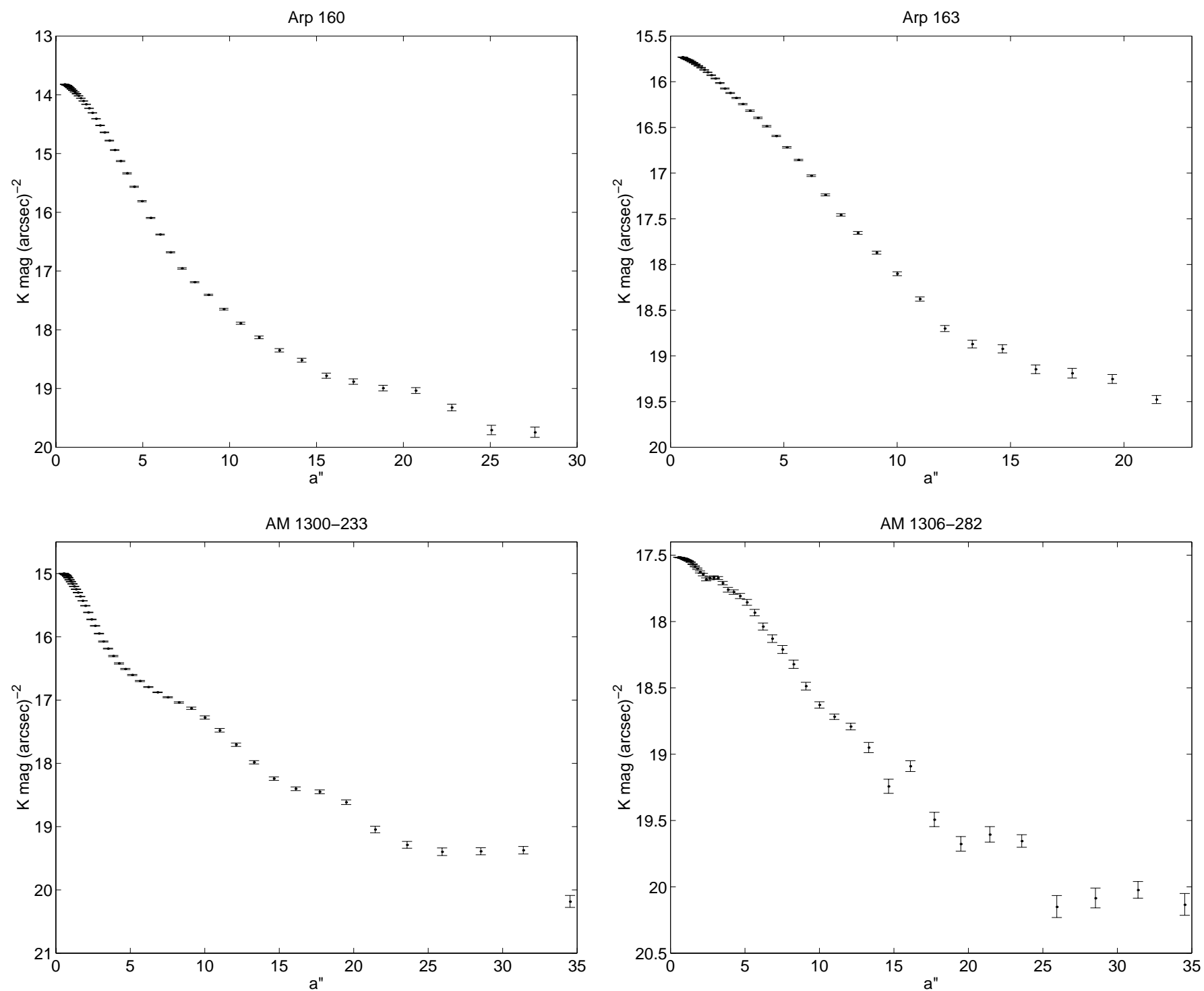

Fig. 4. Galaxies that could not be fit. The $K_{\mathrm{s}}$ band magnitude in mag $\operatorname{arcsec}^{-2}$ is plotted against the semi-major axis, $a^{\prime \prime}$. 


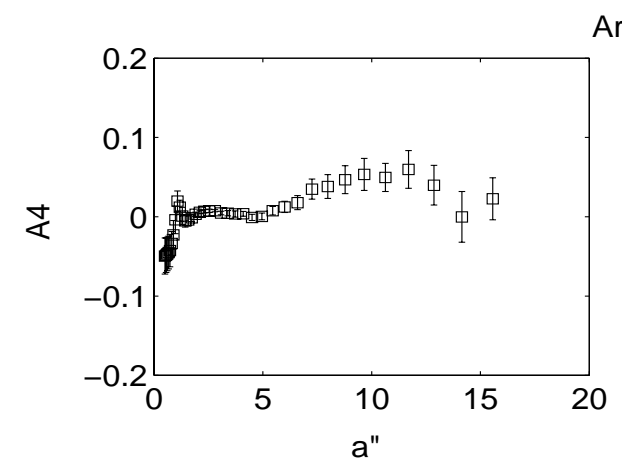

Arp 193
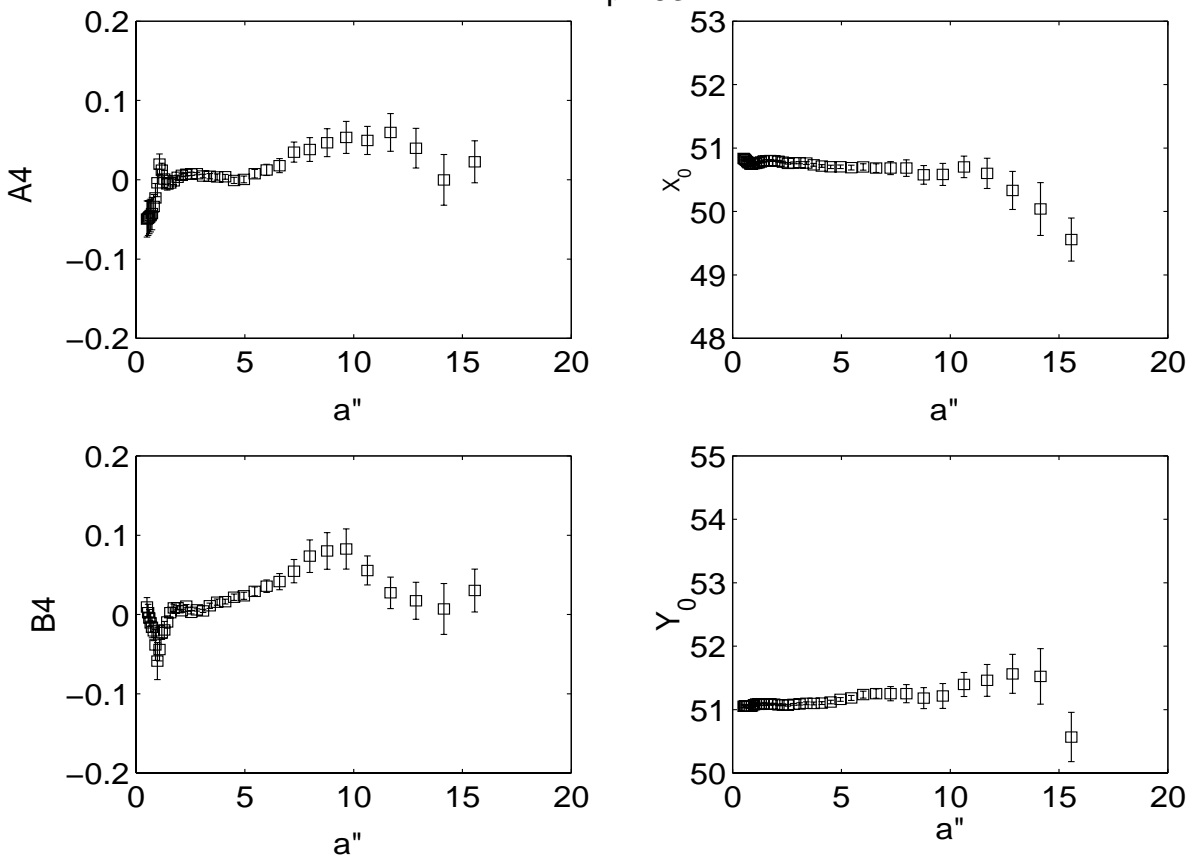

Arp 163
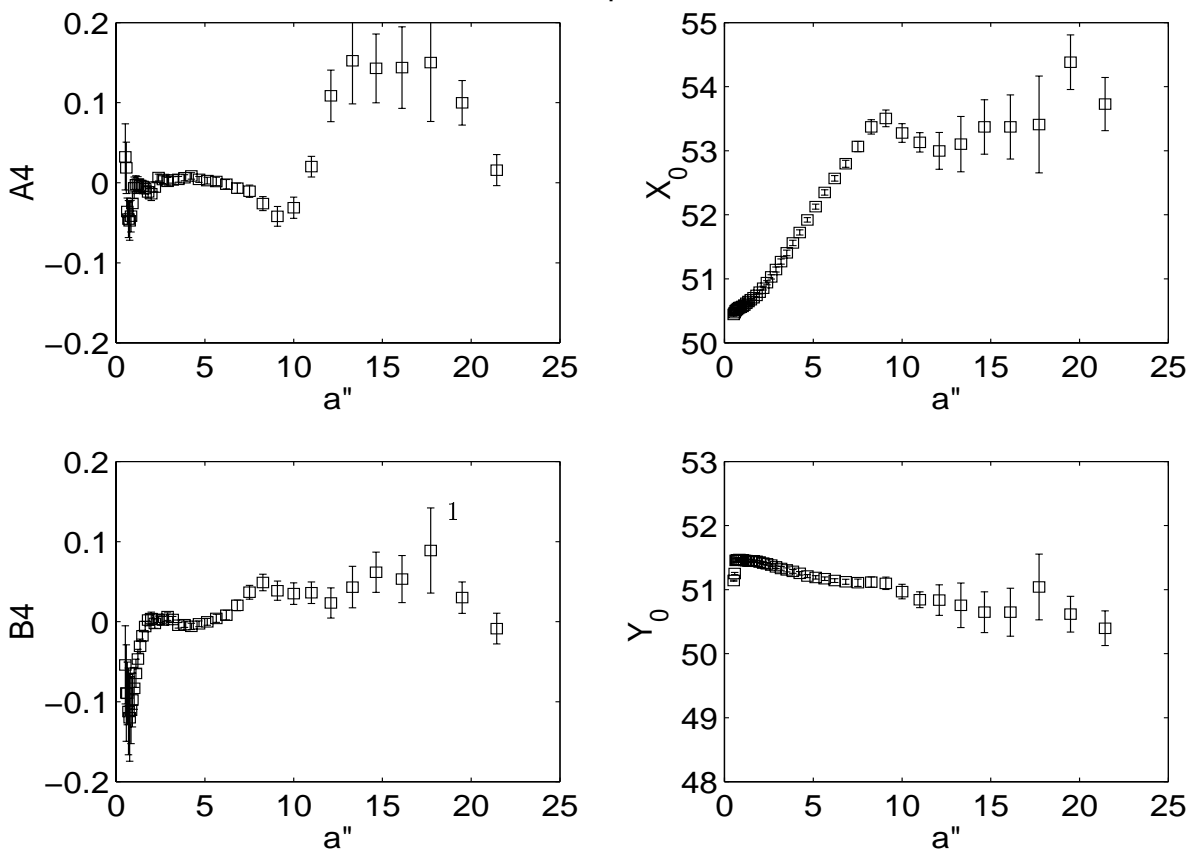

Fig. 5. $A 4, B 4, X 0$ and $Y 0$ for the Class I galaxy Arp 193 and the unrelaxed galaxy Arp 163. The inner isophotes have a common centre for the relaxed system, Arp 193, whereas in contrast, the centre shows a wandering or a sloshing behaviour for the unrelaxed system Arp 163.

and the photometric properties of the expected remnants. They study the distribution of the relative position angle $(\Delta \phi)$ between the isophote at $0.5 r_{\text {eff }}$ and $1.5 r_{\text {eff }}$ versus the effective ellipticity $\epsilon_{\text {eff }}$ (ellipticity at $1.5 r_{\text {eff }}$ ), where $r_{\text {eff }}$ is the half-light radius, for random projections of each merger remnant. Since our sample may cover different mass ratios viewed at different inclination angles, it is meaningful to compare the observed values of $(\Delta \phi)$ and $\epsilon_{\text {eff }}$ with their results to see if there is an overlap. We use the half-light radii derived from the elliptical aperture photometry of our sample and plot the behaviour of $\Delta \phi$ versus $\epsilon_{\text {eff }}$ for our sample of galaxies in Fig. 7. We find that our observed values lie in the same region of the plot as predicted theoretically by them. However, since most of the region is common to all the four mass ratios, no clear comment can be made about the mass ratios of the progenitors. Nevertheless, what is interesting is that a few of our objects clearly lie in the region corresponding to unequal mass mergers. 

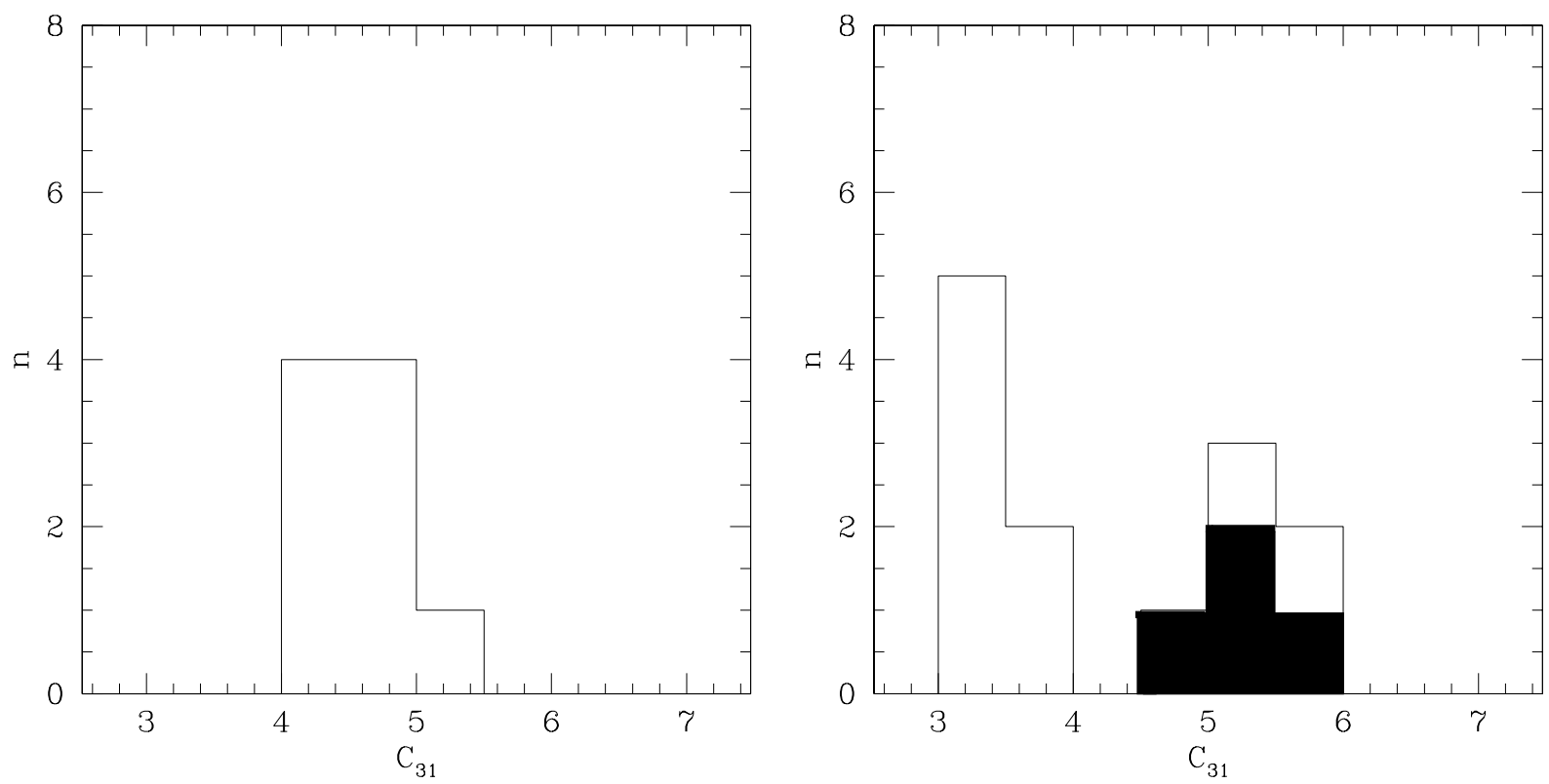

Fig. 6. Distribution of the number of galaxies versus the concentration indices $C_{31}$ of the sample. The figure on the left denotes Class I objects and the figure on the right denotes Class II. The shaded region comprises of objects which are AGN/LINERs.

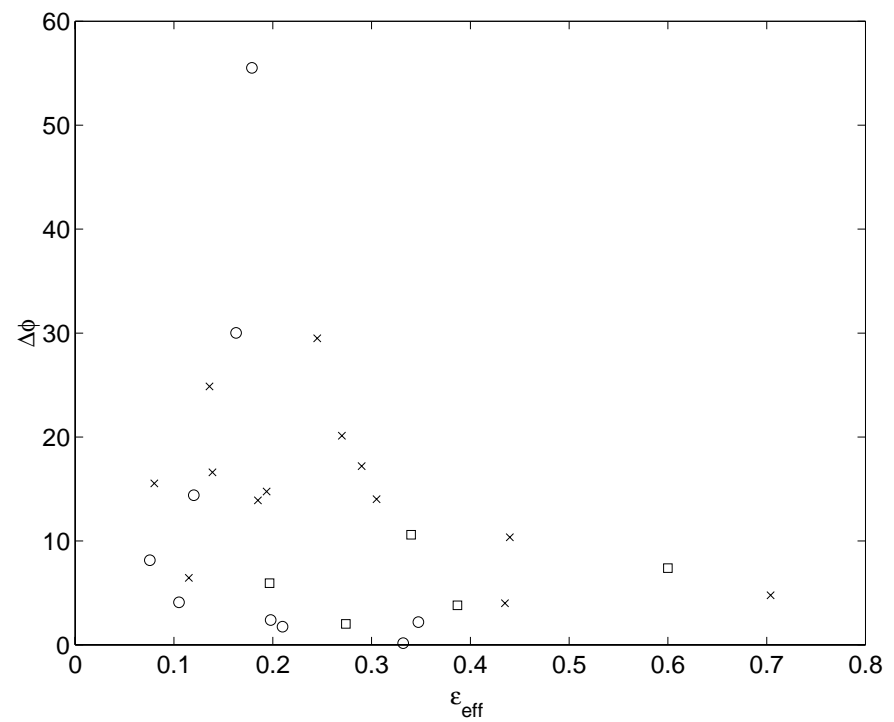

Fig. 7. Distribution of the relative position angle $\Delta \phi$ between $0.5 r_{\text {eff }}$ and $1.5 r_{\text {eff }}$ with the ellipticity $\epsilon_{\text {eff }}$ at $1.5 r_{\text {eff }}$. Class I objects are denoted by circles, Class II by crosses and the unrelaxed objects by squares. Our observed values lie in the same region of the plot as predicted theoretically for mergers by Naab \& Burkert (2001).

\subsection{Other properties}

Interestingly, the various classes in our study do not show any clear demarcation in properties like $F(100 \mu) / F(60 \mu)$ or the infrared luminosity. The ratio of the infrared luminosity to the molecular hydrogen mass (taken from Georgakakis et al. 2000) does not also show any clear demarcation between the classes. Also, there is no correlation between the class to which the galaxy belongs and the blue luminosities or the $K_{\mathrm{s}}$ band magnitudes. Therefore, it is the ratio of the galaxy masses that seems to be the main parameter that decides the class to which a merger remnant belongs.

Some hints about the physical differences between these two classes could be provided by the HI distribution in these systems since it traces the dynamical consequences of encounters at large radii. Hibbard (2001) has compiled HI maps for peculiar galaxies and galaxies showing peculiar HI distributions and divided them into various groups. An inspection of these maps shows that seven of the objects from our sample are included. Interestingly, the three objects from Class I namely Arp 193, Arp 226 and Arp 243 all fall in the same group of interacting doubles made up of two HI systems with two HI tails. Four objects in Class II fall in different groups. Arp 186 is classified as interacting double made up of two HI systems with one HI tail; Arp 214 has a two-sided HI warp, Arp 224 as interacting doubles made up of two systems with only one containing HI and Arp 230 is a peculiar early type galaxy with HI within the optical body. This evidence points to a homogeneous set of initial conditions as indicated by similar HI properties for Class I. In contrast, Class II objects show a diversity of HI behaviour, which points to varied initial conditions for the galaxy encounter or with varied parameters for the progenitor galaxies.

\subsection{Comparison with earlier work}

SB91 carried out a study similar to our work, namely a near infrared study of advanced disk-disk mergers of galaxies chosen from the Arp sample. Six galaxies from their sample overlap with our sample. However, they computed and fit the surface brightness profiles only along the major axis and the minor axis and studied their deviations from the $r^{1 / 4}$ law along these two axes. Our study consists of 
fitting ellipses to the galaxy image and deriving an averaged profile. Thus, our study gives a better sampling of the galaxy brightness distribution. We find that out of the six galaxies in common, five can be fit by an $r^{1 / 4}$ profile while one galaxy, namely Arp 224, is fit better by an outer exponential.

Reshetnikov et al. (1993) studied a sample of 73 galaxies in close interacting systems selected from the Arp catalogue in the $R$ band. Their sample concentrated on close double or triple interacting systems and was selected based on the presence of obvious morphological signs of interactions, such as bridges, tails, distortions, envelopes, etc. They found that 10 galaxies show an $r^{1 / 4}$ profile, while 28 have pure exponential brightness distributions and 30 galaxies show both components. They report that bulges of interacting elliptical and S0 galaxies are more compact compared to normal galaxies, while bulges of interacting spirals are similar to those of non-interacting galaxies. Our study concentrates on advanced merger candidates where the systems have merged to a single nucleus stage. Hence, we are sampling a more advanced stage in the merger process compared to the Reshetnikov sample.

Zheng et al. (1999) studied a sample of 13 singlenucleus ultraluminous galaxies using HST images. They find a nearly equal number of galaxies that can be fitted by an $r^{1 / 4}$, inner $r^{1 / 4}$ plus an outer extension and profiles that deviate from the $r^{1 / 4}$ law. Scoville et al. (2000) studied a sample of 24 luminous infrared galaxies and found that 9 of them could be approximately fit by a $r^{1 / 4}$ law. They also report that a majority of the systems (13 out of 24) could be fit equally well by either an $r^{1 / 4}$ or an exponential law. The result from our paper, namely that a merger need not always lead to a luminosity profile like that of elliptical galaxies, is consistent with the results obtained by these authors, except for a different sample.

We studied the distribution of the concentration index $C_{31}$ with $M_{K_{\mathrm{s}}}$ (the absolute $K_{\mathrm{s}}$ magnitude) (Fig. 8) and found that all the galaxies that can be fit by an $r^{1 / 4}$ law are brighter than $-21.0^{\mathrm{m}}$ and have a concentration index greater than 3.5. We also find that there is a trend for lower luminosity galaxies to have smaller values of $C_{31}$. A similar study by Boselli et al. (1997) of Virgo cluster galaxies showed that $C_{31} \leq 3$ for all galaxies fainter than $10.0^{\mathrm{m}}$ in $K^{\prime}$, independent of the morphological type of the galaxy. They argue that this is consistent with an exponential disk. Similarly, Gavazzi et al. (1996) found that luminous galaxies are more centrally concentrated.

\section{Conclusions}

A study of a sample of morphologically selected disturbed galaxies with indications of mergers shows that in spite of their similar optical appearances, the luminosity and hence mass profiles of these galaxies fall into two distinct classes. Thus, the dynamical evolution is distinctly different in the two classes. Class I is made up of galaxies that are fit by an $r^{1 / 4}$ law and hence are completely relaxed systems. Class II objects show the presence of an exponential

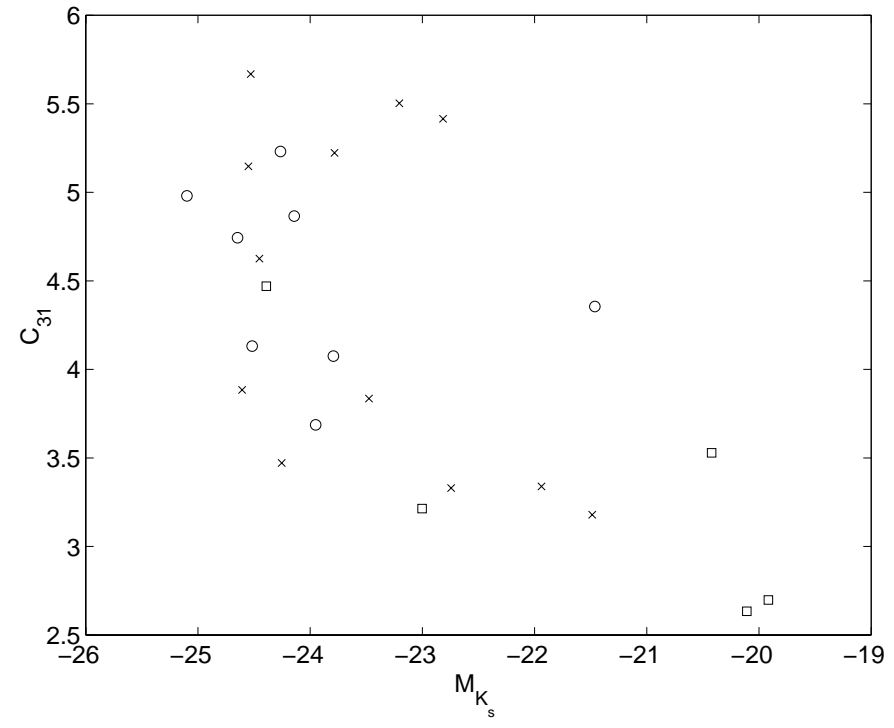

Fig. 8. The concentration index as a function of the absolute $K_{\mathrm{s}}$ magnitude $\left(M_{K_{\mathrm{s}}}\right)$. The open circles denote Class I, the crosses denote Class II and the squares denote the unrelaxed objects.

disk in their outer regions. A few objects cannot be fit by either law. Thus, the dynamical properties of these classes are distinct. These classes do not show any differences in various properties, such as IR luminosities, molecular gas content or the IR colours. We find that our sample of morphologically disturbed galaxies may be merger products in spite of not being bright in the IR. Class I objects are likely to have formed as a result of merging of equal mass galaxies and have relaxed completely. Class II objects are likely to be the result of unequal mass mergers in which the exponential disk of the primary progenitor has survived. The unrelaxed objects are likely to be recent mergers.

We have also studied the structural parameters such as $B 4$ and the location of the centre of mass, that provides important information on mergers, in addition to the mass distribution as indicated by the luminosity profiles. It is seen that the concentration index $C_{31}$ for Class I objects shows a maximum between 4 and 5 . For Class II objects, there is a clear demarcation between the active and the non-active galaxies. The active galaxies show a peak between 5 and 5.5 while the non-active galaxies show a peak between 3 and 3.5. Thus, it is seen that active galaxies with an exponential disk are more centrally concentrated than completely relaxed systems which in turn are more centrally concentrated than non-active galaxies with an outer exponential disk.

In summary, 13 out of 27 disturbed galaxies with indications of mergers are found to exhibit exponential disk behaviour. Hence, the existence of this class is a robust result and this class of galaxies requires further dynamical study. 
Acknowledgements. We are very thankful to the referee, R. A. Koopmann for detailed and constructive comments which have greatly improved the paper.

This publication makes use of data products from the Two Micron All Sky Survey, which is a joint project of the University of Massachusetts and the Infrared Processing and Analysis Center/California Institute of Technology, funded by the National Aeronautics and Space Administration and the National Science Foundation. This research has made use of the NASA/ IPAC Infrared Science Archive, which is operated by the Jet Propulsion Laboratory, California Institute of Technology, under contract with the National Aeronautics and Space Administration, the NASA/IPAC Extragalactic Database (NED) which is operated by the Jet Propulsion Laboratory, California Institute of Technology, under contract with the National Aeronautics and Space Administration, the SIMBAD database, operated at CDS, Strasbourg, France, the LEDA and the HYPERCAT databases and NASA's Astrophysics Data System Bibliographic Services.

\section{Appendix A: Notes on individual galaxies}

The structural parameters deduced for the galaxies in our sample (Sect. 3.3) are listed below:

Arp 156: low ellipticity, isophotal twisting.

Arp 165: slightly disky, nearly constant PA.

Arp 193: disky throughout, nearly constant PA.

Arp 221: inner disky, outer boxy between $10^{\prime \prime}$ and $20^{\prime \prime}$.

Arp 222: disky up to $30^{\prime \prime}$ (between 0.6 to $3.7 \mathrm{kpc}$ ), outer boxy.

Arp 225: no clear trends, nearly constant PA.

Arp 226: very disturbed profile.

Arp 231: boxiness beyond $8^{\prime \prime}$ (between 2.9 and

$5.8 \mathrm{kpc})$, PA keeps changing erratically.

Arp 243: inner disky, outer boxy (beyond $2.1 \mathrm{kpc}$ ). centre starts changing beyond $2.1 \mathrm{kpc}$.

Arp 162: disky beyond $15^{\prime \prime}(1.3 \mathrm{kpc})$, nearly constant PA.

Arp 186: strong isophotal twisting, inner disky (up to

$2.4 \mathrm{kpc}$ ), outer boxy. in PA.

Arp 18\%: slightly disky throughout, small changes

Arp 212: disky, changing centre, isophotal twisting.

Arp 214: disky, erratically changing centre.

Arp 219: inner disky (between 2.6 and $4.7 \mathrm{kpc}$ ), boxy beyond.

Arp 224: isophotal twisting, erratic $B 4$.

Arp 230: disky up to about $1.9 \mathrm{kpc}$, isophotal twisting. $A M$ 038-230: inner boxy (up to $4.8 \mathrm{kpc}$ ), outer disky

(between 4.8 and $8.9 \mathrm{kpc}$ ), isophotal twisting.

AM 0337-312: inner boxy (between 2.9 and $5.2 \mathrm{kpc}$ ), highly inclined, nearly constant PA.

$A M$ 1324-431: inner boxy.

$A M$ 1315-263: isophotal twisting, boxy beyond $25^{\prime \prime}$.

$A M$ 2146-350: inner boxy, outer disky, isophotal twisting.

$A M$ 0501-632: disky, isophotal twisting. The only galaxy fit by an exponential throughout.
Arp 160: inner disky, outer boxy, continuously changing centre.

Arp 163: disky, continuously changing centre.

AM 1300-233: inner disky, outer boxy, changing centre. AM 1306-282: disky up to $10^{\prime \prime}$, changing centre.

\section{References}

Arp, H. C. 1966, Atlas of Peculiar Galaxies (Pasadena: California Institute of Technology)

Arp, H. C., \& Madore, B. F. 1987, A Catalog of Southern Peculiar Galaxies and Associations (Cambridge Univ. Press, Cambridge)

Baggett, W. E., Baggett, S. M., \& Anderson, K. S. J. 1998, AJ, 116, 1626

Barnes, J. E. 1988, ApJ, 331, 699

Barnes, J. E. 1998, in Interactions and Induced Star Formation: Saas-Fee Advanced Course 26, ed. D. Friedli et al. (Berlin: Springer Verlag), 275

Barnes, J. E., \& Hernquist, L. E. 1991, ApJ, 370, L65

Bekki, K. 1998a, ApJ, 496, 713

Bekki, K. 1998b, ApJ, 502, L133

Binney, J., \& Merrifield, M. 1998, Galactic Astronomy (Princeton: Princeton Univ. Press)

Boselli, A., Tuffs, R. J., Gavazzi, G., Hippelein, H., \& Pierini, D. 1997, A\&AS, 121, 507

de Vaucouleurs, G. 1977, in Evolution of Galaxies and Stellar Populations, ed. B. M. Tinsley, \& R. B. Larson (New Haven: Yale University Obs.), 43

Gavazzi, G., Pierini, D., \& Boselli, A. 1996, A\&A, 312, 397

Georgakakis, A., Forbes, D. A., \& Norris, R. P. 2000, MNRAS, 318,124

Hernquist, L. E. 1992, ApJ, 400, 460

Hernquist, L., \& Spergel, D. N. 1992, ApJ, 399, L117

Hibbard, J. E., van Gorkom, J. H., Rupen, M. P., \& Schiminovich, D. 2001 [astro-ph/0110667]

James, P., Bate, C., Wells, M., Wright, G., \& Doyon, R. 1999, MNRAS, 309, 585

Jedrejewski, R. I. 1987, MNRAS, 226, 747

Joseph, R. D., \& Wright, G. S. 1985, MNRAS, 214, 87

Kenney, J. D. P., Koopmann, R. A., Rubin, V. C., \& Young, J. S. 1996, AJ, 111, 152

Lu, N. Y. 1998, ApJ, 506, 673

Lynden-Bell, D. 1967, MNRAS, 136, 101

Naab, T., \& Burkert, A. 2001 [astro-ph/0110179]

Nieto, J.-L., \& Bender, R. 1989, A\&A, 215, 266

Reshetnikov, V. P., Hagen-Thorn, V. A., \& Yakovleva, V. A. 1993, A\&AS, 99, 257

Sanders, D. B., \& Mirabel, I. F. 1996, ARA\&A, 34, 749

Schweizer, F. 1996, AJ, 111, 109

Schweizer, F. 1982, ApJ, 252, 455

Scoville, N. Z., Evans, A. S., Thompson, R., et al. 2000, AJ, 119,991

Stanford, S. A., \& Bushouse, H. A. 1991, ApJ, 371, 92

Toomre, A. 1977, in The Evolution of Galaxies and Stellar Populations, ed. B. M. Tinsley, \& R. B. Larson (New Haven: Yale University Obs.), 401

van Albada, T. S. 1982, MNRAS, 201, 939

Walker, I. R., Mihos, J. C., \& Hernquist, L. 1996, ApJ, 460, 121

Wright, G. S., James, P. A., Joseph, R. D., \& McLean, I. S. 1990, Nature, 344, 417

Zheng, Z., Wu, H., Mao, S., et al. 1999, A\&A, 349, 735 